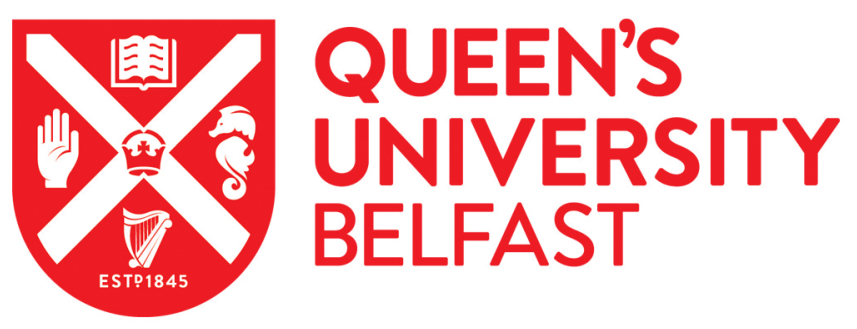

\title{
Accurate and Efficient Modelling to Calculate the Voltage Dependence of Liquid Crystal Based Reflectarray Cells
}

Perez-Palomino , G., Florencio, R., Encinar, J. A., Barba, M., Dickie, R., Cahill, R., Baine, P., Bain, M., \& Boix, R. R. (2014). Accurate and Efficient Modelling to Calculate the Voltage Dependence of Liquid Crystal Based Reflectarray Cells. IEEE Transactions on Antennas and Propagation, 62(5), 2659-2668.

https://doi.org/10.1109/TAP.2014.2308521

Published in:

IEEE Transactions on Antennas and Propagation

Document Version:

Peer reviewed version

Queen's University Belfast - Research Portal:

Link to publication record in Queen's University Belfast Research Portal

Publisher rights

(c) 2015 IEEE. Personal use of this material is permitted. Permission from IEEE must be obtained for all other users, including reprinting/ republishing this material for advertising or promotional purposes, creating new collective works for resale or redistribution to servers or lists, or reuse of any copyrighted components of this work in other works

\section{General rights}

Copyright for the publications made accessible via the Queen's University Belfast Research Portal is retained by the author(s) and / or other copyright owners and it is a condition of accessing these publications that users recognise and abide by the legal requirements associated with these rights.

Take down policy

The Research Portal is Queen's institutional repository that provides access to Queen's research output. Every effort has been made to ensure that content in the Research Portal does not infringe any person's rights, or applicable UK laws. If you discover content in the Research Portal that you believe breaches copyright or violates any law, please contact openaccess@qub.ac.uk. 


\title{
Accurate and Efficient Modeling to Calculate the Voltage Dependence of Liquid Crystal Based Reflectarray Cells
}

\author{
Gerardo Perez-Palomino, Rafael Florencio, José A. Encinar, Fellow, IEEE, Mariano Barba, Member, \\ IEEE, Raymond Dickie, Robert Cahill, Senior Member, IEEE, Paul Baine, Michael Bain, \\ and Rafael R. Boix, Member, IEEE,
}

\begin{abstract}
Two models which can predict the voltage dependent scattering from Liquid Crystal (LC) based reflectarray cells are presented. The validity of both numerical techniques is demonstrated using measured results in the frequency range 94 $110 \mathrm{GHz}$. The most rigorous approach models, for each voltage, the inhomogeneous and anisotropic permittivity of the LC as a stratified media in the direction of the biasing field. This accounts for the different tilt angles of the LC molecules inside the cell calculated from the solution of the elastic problem. The other model is based on an effective homogeneous permittivity tensor that corresponds to the average tilt angle along the longitudinal direction for each biasing voltage. In this model, convergence problems associated with the longitudinal inhomogeneity are avoided and the computation efficiency is improved. Both models provide a correspondence between the reflection coefficient (losses and phase-shift) of the LC-based reflectarray cell and the value of biasing voltage, which can be used to design beam scanning reflectarrays. The accuracy and the efficiency of both models are also analyzed and discussed.
\end{abstract}

Index Terms - Reconfigurable reflectarray, liquid crystal (LC), molecular tilt angle, anisotropy, longitudinal inhomogeneity, effective homogeneous tensor, effective permittivity.

\section{INTRODUCTION}

$\mathrm{T}$ HE use of liquid crystals (LCs) to provide tunable devices and reconfigurable antennas operating at millimeter and sub-millimeter wavelengths has recently been investigated [1][8]. The role played by the LC in these devices is to provide a tunable permittivity layer when a quasi-static bias voltage is applied. The principle of operation of a LC-based reflectarray antenna involves changing the resonant frequencies (and the phase-shift) of the printed conductive patches which are placed above a grounded LC-substrate, by varying the bias voltage applied to each element in the array [5]-[6]. Thus, to obtain a certain radiation pattern, the LC in each cell that composes the reflectarray antenna must be biased with the corresponding

Manuscript received June 5, 2013. This work was supported in part by the European Space Agency, under project 4000103061, and by the Spanish Ministry of Science and Innovation, under the projects TEC2010-17567 and CSD2008-00068 (Consolider-Ingenio 2010).

G. Perez-Palomino, J. A. Encinar, and M. Barba are with the Department of Electromagnetism and Circuit Theory, Universidad Politécnica de Madrid, E28040, Madrid, Spain (e-mail: gperez@etc.upm.es). voltage that provides the required phase-shift [6]-[8].

In [8], the extreme phase states, associated with the voltage values $\mathrm{V}=0$ and the saturation voltage $(\mathrm{V}>>\mathrm{Vth}$; $\mathrm{Vth}$ is the Freederiks threshold voltage), were accurately modeled considering the anisotropy of the LC-cell. The phase response versus frequency for these two states was used to design reflectarray cells which exhibited improved performance. For both unbiased and saturated voltages, the orientation of the LC molecules does not vary along $\mathrm{z}$, which is the direction of the biasing field; however this is not the case for the intermediate states. Although a homogeneous and isotropic effective permittivity $\left(\varepsilon_{e f f}(V)\right)$ was also defined in [8] for describing the LC layer at the intermediate biasing states, the reflection coefficient (and the effective permittivity) has only been related at present with the bias voltage at the extremes states, because of the difficulties in modeling the anisotropy and inhomogeneity in the direction of the biasing field for the intermediate states in resonant reflectarray cells. Since the design of a reflectarray antenna requires defining all the biasing voltages to provide the required phase-shift in each element, it can only be accomplished after measuring the curves, PhaseVoltage (Ph-V).

Since the reflection coefficient of the cells generally depends on the angle of incidence, the cell dimensions and frequency [8], the voltage dependence (not linear) must be measured at several angles and frequencies for a given antenna configuration. The large number of measurements required to characterize the LC-reflectarray cells makes the acquisition of data very complicated, costly and often unaffordable. If the cells are designed to exhibit phase linearity in the operating band and low sensitivity to the angle of incidence (the cells are dimensionally equal along the antenna), the acquisition of measured data is simplified because the $\mathrm{Ph}-\mathrm{V}$ curves could be measured at only one angle of incidence and at one frequency for each bias voltage [8]. However, even in this case, the procedure is adequate only if some phase errors are acceptable.

R. Florencio, and R.R. Boix, are with Microwaves Group, Department of Electronics and Electromagnetism, College of Physics, University of Seville, Avda. Reina Mercedes s/n, 41012-Seville, Spain (e-mails: rflorencio@us.es).

R. Dickie, R. Cahill, M. Bain, and P. Baine, are with the Institute of Electronics, Communications and Information Technology, Queen's University Belfast, Belfast BT3 9DT, Northern Ireland, UK,. (e-mail: r.cahill@qub.ac.uk) 
These errors, which can be up to 60 degrees in the worst case for multi-resonant cells, can have a little effect on the radiation patterns for certain antenna configurations. This is the case for beam scanning antennas with a single reflectarray, where the phase error is of same order as the quantization error in 2-bit phase-shifters. However, other applications require more accurate beam-forming, or the use of antenna configurations that are very sensitive to phase variations so that these errors produce significant distortion of the radiation patterns.

Therefore, a numerical tool that allows the voltage dependence of LC-reflectarray cells to be calculated at different angles of incidence and frequencies with good accuracy is necessary, in order to obtain the required bias voltages that should be applied to each cell to reconfigure or to scan the beam.

In the work reported in this paper, two procedures to calculate the voltage dependence of Liquid Crystal-based reflectarray cells are proposed and validated using experimental data. Firstly, accurate modeling of the intermediate biasing states in LC's that takes into account the anisotropy and inhomogeneity in the direction of the biasing field (longitudinal inhomogeneity) is applied and evaluated for first time for reflectarray cells. The inhomogeneous permittivity tensor is obtained for each voltage from the solution of the elastic problem related to the biasing of the LC [9]-[14]. Then, a stratified medium, which is derived from the segmentation of the corresponding tensor at each voltage, is used to model the LC and to calculate the reflection coefficient by using an electromagnetic simulator based on Spectral-Domain Method of Moments (SD-MoM), which in turns allows the extraction of the voltage dependence of the reflectarray cells. The convergence and the number of layers that are required to model each one of the intermediate states are discussed.

Since the stratified model requires segmenting the LC into a large number of thin layers, problems related to the convergence and complexity in the calculus are shown to occur, thus making the process inefficient. To solve these problems, the use of an effective homogeneous permittivity tensor for each voltage is also proposed. The capabilities of these effective tensors to model the intermediate states and therefore to efficiently predict the voltage dependence of the reflectarray cells at an arbitrary angle of incidence and/or frequency is analyzed and experimentally evaluated. This technique provides efficient computation of the biasing voltages required to scan or reconfigure a beam in LC-based reflectarrays.

\section{Static Modeling OF LiQuid CRYSTALS IN THE NEMATIC PHASE}

The tunable permittivity of the LC at macroscopic level is derived from the physical properties of the LC molecules. The nematic phase of LC material is characterized by having a relatively large degree of orientational order. Since the LC is usually formed by rod-like molecules, all molecules have their axes locally oriented in the same average direction, so that the LC is locally uniaxial and homogeneous, and presents macroscopically two permittivities: $\varepsilon_{/ /}$(in the direction of the axes) and $\varepsilon_{\perp}$ (in the directions orthogonal to the axes). On the other hand, the LC is also characterized by having a relatively short degree or positional order, so the molecules are able to rotate under certain external excitations.

If a LC medium at a certain frequency and temperature (guaranteeing the nematic state) is considered, and an arbitrary Cartesian coordinate system is fixed, the relative permittivity tensor can be written by [9]:

$$
\overline{\overline{\varepsilon_{r}}}(\bar{r})=\varepsilon_{r \perp} \overline{\bar{I}}+\Delta \varepsilon_{r} \overline{\bar{N}}(\bar{r})
$$

where $\overline{\bar{I}}$ is the second order identity tensor whose components are the elements of the $3 \times 3$ identity matrix, $\Delta \varepsilon_{r}=\varepsilon_{r / /}-\varepsilon_{r \perp}$ is the dielectric anisotropy, and $\overline{\bar{N}}(\bar{r})$ is the second order tensor given by the tensor product of the vector $\hat{n}(\bar{r})$ with itself, $\overline{\bar{N}}(\bar{r})=\hat{n}(\bar{r}) \otimes \hat{n}(\bar{r})$. The vector $\hat{n}(\bar{r})$ is a unit vector that macroscopically defines the local average direction of the molecules in a small volume as a function of the position (the director) in the chosen coordinate system. Therefore, if an external excitation produces a rotation of the vector $\hat{n}$, the permittivity will vary.

Usually, the excitation of LC is produced by applying a quasistatic electric field, although a magnetic field could also be used. If LC in the nematic state is confined in a finite volume, $V_{l c}$, in which a quasi-static electric field is applied, the electrostatic energy is converted into elastic energy so that the material is deformed ( $\hat{n}$ varies). To calculate how the director has varied when all the energy has been transformed (static or equilibrium point), a free energy functional, $F$, is minimized:

$$
\min (F)=\min \left(\iiint_{V l c}\left(g_{v}-g_{q}\right) d V_{l c}+\oiint_{S} g_{s} d S\right)
$$

where $g_{v}$ and $g_{q}$ are the Frank-Oseen elastic and electrostatic free energy densities:

$$
\begin{aligned}
& g_{v}=\frac{k_{11}}{2}(\nabla \cdot \hat{n})^{2}+\frac{k_{22}}{2}(\hat{n} \cdot \nabla \times \hat{n})^{2}+\frac{k_{33}}{2}|\hat{n} \times(\nabla \times \hat{n})|^{2} \\
& g_{q}=\frac{1}{2} \varepsilon_{0} \overline{E_{q}} \cdot \underbrace{\left(\varepsilon_{r q \perp} \overline{\bar{I}}+\Delta \varepsilon_{r q} \overline{\bar{N}}\right)}_{\overline{\sigma_{r q}}} \cdot \overline{E_{q}}
\end{aligned}
$$

and $g_{s}$ is the free energy density related to the effect of the anchoring produced at the orienting surface that impose certain boundary condition to the molecules in the surface, $\mathrm{S}$, that encloses the volume [9]. The parameters $k_{11}, k_{22}, k_{33}$ are the elastic constant associated to bend, twist and splay deformations, $\Delta \varepsilon_{r q}=\varepsilon_{r q / /}-\varepsilon_{r q \perp}$ is the LC dielectric anisotropy at the quasi-static excitation frequency and $\overline{E_{q}}$ is the quasi-static electric field applied.

Numerical methods have been used to solve (2) for LC-devices with different geometries and the corresponding boundary conditions [10]-[11]. However, if the LC is confined by two parallel plates separated a distance $z=d$ on which orienting layers are placed (orienting layers impose molecular orientations in the $\mathrm{YZ}$ plane), and if the LC is excited by a 


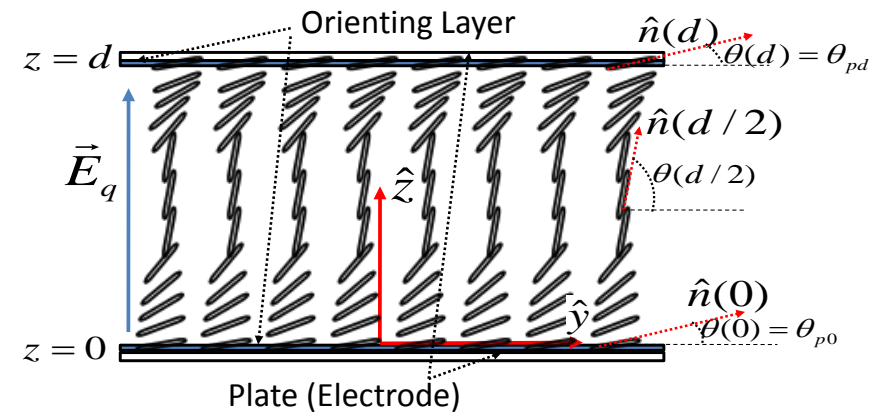

Fig. 1. Molecular orientation in a LC-layer confined between two parallel plates and orienting layers, excited by a homogeneous quasi-static electric field.

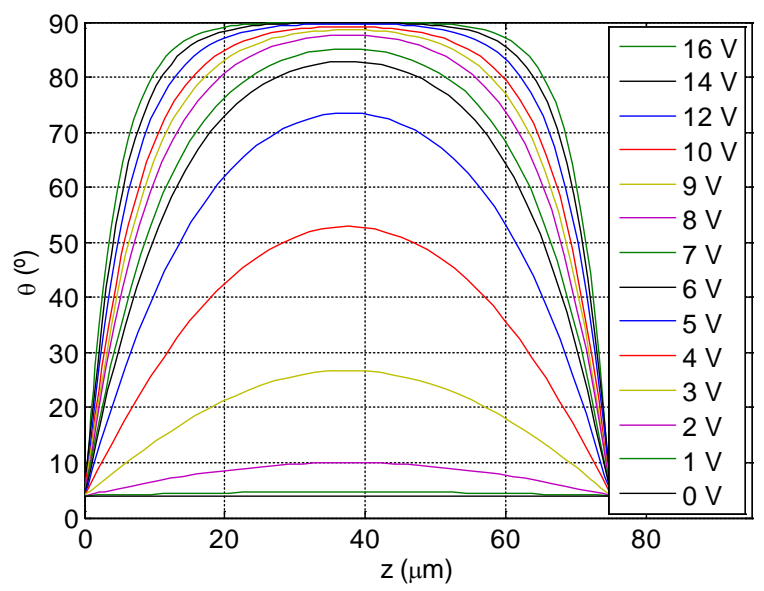

Fig. 2. Tilt angle of the LC-molecules $(\theta)$ as a function of the distance $(z)$ for several RMS voltages in a 75- $\mu \mathrm{m}$ thick LC-layer (LC GT3-23001, $\left.\varepsilon_{r q / /}(1 \mathrm{kHz})=8.1, \Delta \varepsilon_{r q}(1 \mathrm{kHz})=4.6, k_{11}=24 \mathrm{pN}, k_{33}=34.5 \mathrm{pN}\right)$.

$\theta_{p 0}=\theta_{p d}=4 \stackrel{\circ}{\circ}$

uniform quasi-static electric field with only z-component, $\overline{E_{q}}=$ $E_{q z} \hat{z}$ (see. Fig. 1), the symmetry of the structure makes the molecules rotate in the plane $\mathrm{YZ}$ and $\hat{n}$ can be simplified to be $\hat{n}(z)=\cos (\theta(z)) \hat{y}+\sin (\theta(z)) \hat{z}$, where $\theta$ is the angle between $\hat{n}$ and $\hat{y}$. Under this consideration, assuming that the anchoring at the orienting layers is strong (the term of $g_{s}$ can be neglected), the variational problem (2) (Euler-Lagrange) gives a differential equation in which the function to be obtained is $\theta(z)$ [9]:

$$
\begin{aligned}
& \left(k_{11} \cos ^{2}(\theta)+k_{33} \sin ^{2}(\theta)\right) \frac{d^{2} \theta}{d z^{2}}+\left(k_{33}-k_{11}\right) \\
& \sin (\theta) \cos (\theta)\left(\frac{d \theta}{d z}\right)^{2}+\frac{\varepsilon_{0} \Delta \varepsilon_{r q}}{d^{2}}|V|^{2} \sin (\theta) \cos (\theta)=0
\end{aligned}
$$

being $\mathrm{V}$ the voltage applied, which can be generated by two electrodes $\left(V=E_{q z} d\right)$.

It is assumed in (5) that the RF signal does not influence the director variation. This is a very good approximation because the $\mathrm{RF}$ signal at microwave frequencies generally has an intensity which is too small to excite the molecules.

Equation (5) must be solved assuming the boundary conditions imposed by the two orienting layers at the plates $(z=0, z=$ $d$ ), which usually impose pretilt angles $\left(\theta_{p 0}, \theta_{p d}\right)$ different from zero. Numerical methods to solve ordinary differential equations can be used to solve (5). This equation, or even its general form assuming dynamic behavior (Ericksen-Leslie Equations), has been solved in the field of optics generally with the objective of studying the behavior of LC displays, [12]-[14]. Note that equation (5) assumes that the excitation corresponds with a DC electric field. However, AC excitations can be introduced in the problem by using the concept of root mean square voltage (RMS), and $\varepsilon_{r q / /}, \varepsilon_{r q \perp}$ at the corresponding AC frequency, provided this frequency is much higher than the inverse of the orientation times of the molecules (rise and fall) [14]. Otherwise, the molecular oscillations respect to the equilibrium tilt angle become appreciable, which produces a ripple in both amplitude and phase (AC modulation) that can increase the bit error rate if it is not correctly removed. To avoid this problem, an appropriate selection of the frequency for the bias signal should be made considering the dynamic response of the cells.

As an example that will be used below, Fig. 2 shows the solution of (5) for the structure in Fig.1 at different RMS voltages (at room temperature and $1 \mathrm{kHz}$ AC-signal), assuming a separation of $\mathrm{z}=75 \mu \mathrm{m}$, pretilt angles of $4^{\circ}$ on both surfaces, and LC material type GT3-23001, which is manufactured by Merck for microwave applications. The LC characteristic data given by Merck is shown in the figure caption, and the RF parameters $\left(\varepsilon_{r \perp}\right)$ and $\left(\varepsilon_{r / /}\right)$ have been measured by the authors in the band from 140 to $165 \mathrm{GHz}$ [15]. As can be seen, the molecular tilt angle takes the value $\theta_{p}=4^{\circ}$ at the extremes, increasing its value with the separation until it reaches the maximum at the center of the LC layer. This is because the two orienting layers impose the same pretilt angle. However, for any combination of $\left(\theta_{p 0}, \theta_{p d}\right)$, the curves can be normalized to the distance $\mathrm{d}$. Also, because of the fact that the pretilt angles are different from zero, the molecules will start rotating at voltages below the Frederiks threshold voltage $\left(V_{t h}=\pi\left(k_{11} /\right.\right.$ $\left.\left.\left|\varepsilon_{0} \Delta \varepsilon_{r q}\right|\right)^{1 / 2}\right)$ [14]. It also can be appreciated that the LC is anisotropic and inhomogeneous along $z$ at non-zero voltages. However, both the tilt angle and the homogeneity of the LC (small variation along $\mathrm{z}$ coordinate) increase as the voltage increases, so that the LC can be accurately modeled with a homogeneous anisotropic medium at high voltages near saturation.

Once $\theta(z)$ (and therefore $\hat{n}(z))$ is calculated at a certain voltage, the corresponding $L C$ relative permittivity distribution at a certain RF frequency $\left(f_{R F}\right), \overline{\overline{\varepsilon_{r}}}\left(z, f_{R F}\right)$, can be obtained from (1) using the LC parameters $\left(\varepsilon_{r \perp}\left(f_{R F}\right)\right)$ and $\left(\varepsilon_{r / /}\left(f_{R F}\right)\right)$.

\section{EXtraction of Voltage Dependence of REFLECTARRAY CELLS}

A single-layer LC-based reflectarray cell is shown in Fig. 3. It is composed of a superstrate and a ground plane, forming a cavity that is filled with LC. One or more independent metallized conductors (three parallel unequal length dipoles are shown in Fig. 3) are printed on the lower side of the superstrate 


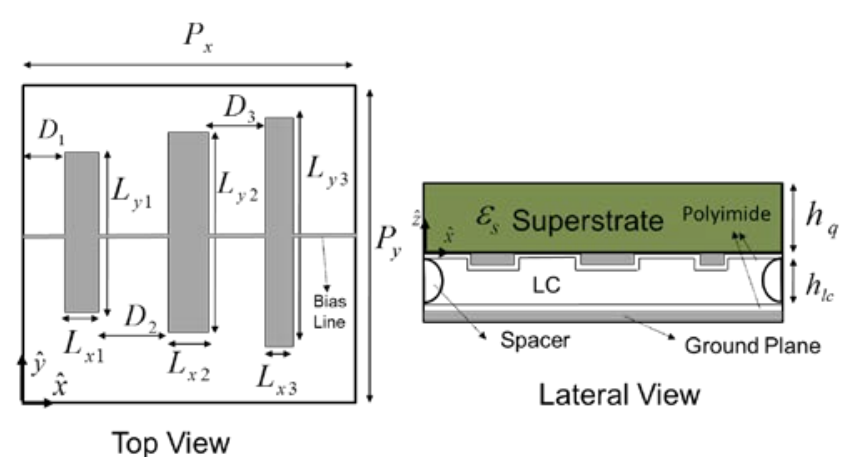

Fig. 3. Single-layer reconfigurable reflectarray cell based on LC with three parallel dipoles for linear polarization. Top and lateral views.

in order to maximize the bandwidth of the structure [16]. Thin rubbed polyimide orienting layers are deposited on the two inner surfaces of the cavity to pre-align the molecules at the repose state $(\mathrm{V}=0)$. The $\mathrm{LC}$ in the cell is biased by applying a voltage between the metallizations (interconnected for the LCbiasing by a thin bias line) and the ground plane, so that the LCexcitation is similar to that shown in Fig. 1, with the exception that the upper electrodes do not cover the entire surface of the cell. This creates transversal inhomogeneity in the LC layer along $x$ and $y$, as a consequence that the quasi-static electric field is not purely polarized in the $\mathrm{z}$ direction throughout the entire LC volume. Consequently, an inhomogeneous tensor along the three dimensions, $\overline{\overline{\varepsilon_{r}}}\left(x, y, z, V, f_{R F}\right)$, is obtained, which may be rigorously calculated by solving (2) with the corresponding quasi-static field (3D LC modeling [10]-[11]).

However, if the transversal inhomogeneity effect is low, the LC can be modelled in the entire cell as an inhomogeneous anisotropic layer in one dimension, $\mathrm{z}$, and the permittivity distribution $\overline{\overline{\varepsilon_{r}}}\left(z, f_{R F}\right)$ can be obtained at each voltage (V) from the elastic problem described in Sec. II, which reduces drastically the problem of modeling and simulating the cells. The effect of the transversal inhomogeneity is usually small in reflectarray cells, since the greatest contribution to the reflection coefficient at the resonance is given by the region under the metallization/s, in which the permittivity tensor has practically only longitudinal variation. Numerical analysis based on the approach described in [17] was used to estimate the maximum phase error obtained if the transversal inhomogeneity is not considered in the model of single resonant cells, which is around $40^{\circ}$ for an extreme case studied. This latter arrangement was for a dipole centered in a cell whose period is much greater than the dipole size, and with a thin LC layer to produce that the gradient of the phase curve around resonance is very steep [16]-[18]. However, if the period of the unit cell is reduced and made similar close to the dipole size, the effect of not considering the transversal inhomogeneity will decrease to a maximum phase error of $15^{\circ}$ or will even be negligible $\left(<1^{\circ}\right)$ if the cell exhibits a smooth phase curve. This can be extended to multi-resonant cells as shown in Fig. 3 (as pointed out in [8]), since the parallel dipoles behave as three independent resonators, or even to other conductor shapes. Thus, reflectarray element geometries with small unit cell
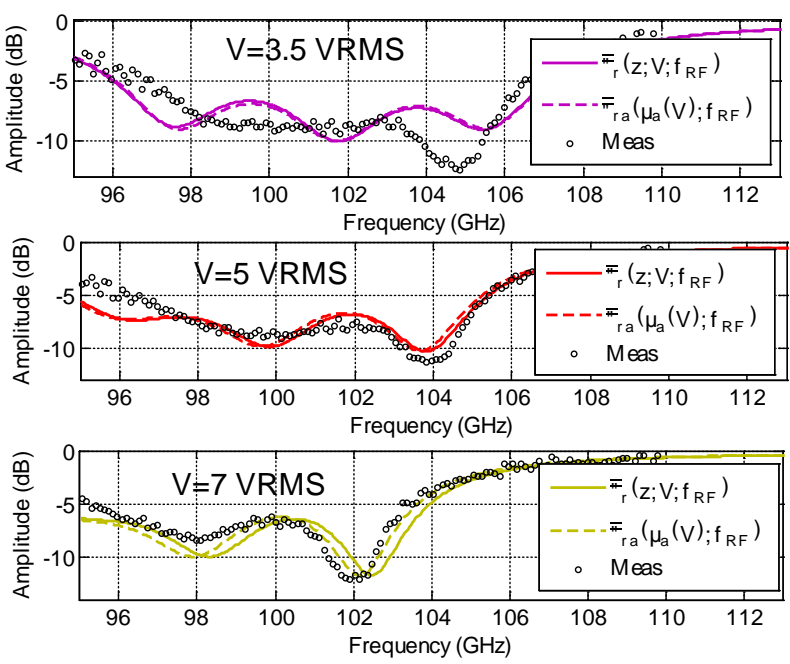

(a)

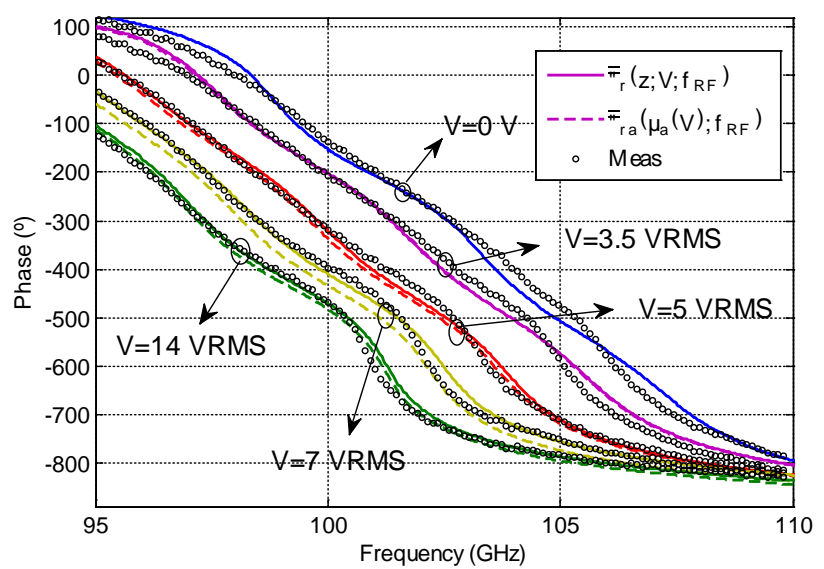

(b)

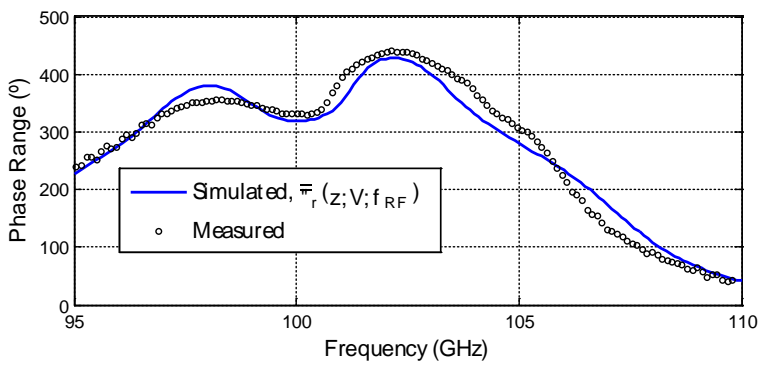

(c)

Fig. 4. Simulated (a) Amplitude, (b) Phase and (c) Phase Range of the reflection coefficient for the reflectarray cell shown in Fig. 3 at different biasing states using accurate modeling $\overline{\overline{\varepsilon_{r}}}\left(z, V, f_{R F}\right)$ and the homogeneous equivalent tensors $\overline{\overline{\varepsilon_{r a}}}\left(\theta_{a}(V), f_{R F}\right)$, superimposed to measurements for TM polarization and incidence $\theta_{\text {in }}=45^{\circ}, \varphi_{\text {in }}=90^{\circ}$. Operating Band: 96 to 104 GHz. Materials: LC GT3-23001 and Quartz as superstrate, $\left(\varepsilon_{\mathrm{s}}=3.78\right.$, $\left.\tan \delta_{\mathrm{s}}=0.002\right)$. Cell dimensions $(\mathrm{mm})$ : $\mathrm{Px}=1.145, \mathrm{Py}=1.093, \mathrm{Ly} 1=0.707$. Ly2 $=0.748$, Ly3 $=0.792, \quad$ Lx1 $=0.20, \quad$ Lx $2=0.211, \quad$ Lx3 $=0.20, \quad$ D $1=0.171$, D2 $=0.096$, D3 $=0.042$, hq $=0.550$, hlc $=0.075$.

periods are insensitive to the effect of the transversal inhomogeneity.

Note that although a maximum error of $40^{\circ}$ is obtained for the case studied, the average error for all the states (which is the 
most important parameter at antenna level) would be around $20^{\circ}$, which can be used in most applications. However, if a very small phase error is required and the cell structure provides a relatively large effect due to the transversal inhomogeneity, this latter can be suppressed by depositing a thin conductive layer (ITO, PDOT) across the surface of the entire cell [15], similar as in LC Displays.

Assuming that the inhomogeneity in the transverse directions is negligible, two strategies to model the intermediate states and extract the voltage versus reflection phase response of the cells are presented below. The first one is based on rigorous modeling of the LC taking into account the inhomogeneity of a stratified anisotropic media along $\mathrm{z}$, whereas the second model uses an effective homogeneous tensor for each voltage.

\section{A. Extraction by using Stratified Media}

Once the inhomogeneous permittivity $\overline{\overline{\varepsilon_{r}}}\left(z, V, f_{R F}\right)$ is obtained, an electromagnetic analysis of the cell must be carried out. This analysis is quite simple at the two extreme states $(V=0$ and $V>>V_{t h}$ ), but is more complicated at the intermediate states because of the inhomogeneity along $z$ (see Fig. 2).

The modeling of the dielectric inhomogeneity makes it necessary to segment the LC layer into an unknown number of layers in the $\mathrm{z}$ direction. Once the segmentation is done, numerical methods that use volume decomposition (FEM, FDTD) can be used with an appropriate formulation that considers anisotropic media and periodic boundary conditions, although the most appropriate method to solve planar periodic cells is the Method of Moments (MoM) [19].

A software tool that implements the Spectral Domain Method of Moments (SD-MoM) based on a multilayered anisotropic Green's function [20]-[22] has been written, and is used to electromagnetically solve the structure shown in Fig. 3, with the LC modeled as an anisotropic stratified media. Entire-domain sinusoidal basis functions have been used to describe the currents on the dipoles, and a surface impedance has been used to consider the conductive losses [19] of the metallic dipoles and ground plane.

Fig. 4 compares the simulated amplitude (a) and the phase (b) of the reflection coefficient as a function of frequency with the measured results of the cell designed, manufactured and measured in a periodical environment in [8] (angle of incidence $\theta_{\text {in }}=45^{\circ}, \varphi_{\text {in }}=90^{\circ}$ and TM polarization) for some biasing voltages. The simulated (and measured) phase range is presented in Fig. 4 (c), in addition to the structural data (according to Fig. 3). The solution of elastic problem associated with the reflectarray cell under study is represented in Fig. 2 (Sec II A), which in turn considers the effects of the orienting layers used in the manufacturing process on the pretilt angle. SE-150 polyimide films (nominal pretilt given by the manufacturer, $4^{\circ}$ ) were used as orienting layers to impose the same boundary conditions on the surfaces $\left(\theta_{p 0}=\theta_{p d}=4^{\circ}\right)$. Note that the rubbings were made in the directions $\overline{d_{r u}}=\hat{y}$ (upper plate) and $\overline{d_{r d}}=-\hat{y}$ (bottom plate) in order to obtain the same pretilt $\left(4^{\circ}\right)$ on both surfaces. Note also that the material was rubbed in the direction that corresponds with the direction

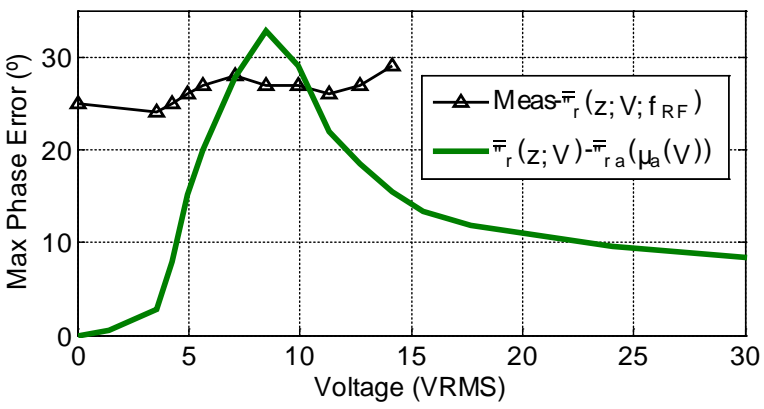

Fig. 5. Maximum Phase Error between measurements and simulations at several voltages using $\overline{\overline{\varepsilon_{r}}}\left(z, V, f_{R F}\right)$. and $\overline{\overline{\varepsilon_{r a}}}\left(\theta_{a}(V), f_{R F}\right)$.

of the incident electric field so that the best electrical performance in the cell of Fig. 3 is obtained (phase-range and losses). If a different direction is chosen, the director would vary in a plane whose normal vector is orthogonal to $\overline{d_{r}}$ and $\hat{z}$ (different from YZ), and the resulting tensors (1) would reduce slightly the performance.

The simulated and measured phase states correspond to the RMS biasing voltages (sinusoidal signal of $1 \mathrm{kHz}$ with no DC offset), $\mathrm{V}=(0 \mathrm{~V}, 3.5 \mathrm{~V}, 5 \mathrm{~V}, 7 \mathrm{~V}, 14 \mathrm{~V})$. The frequency of the sinusoidal bias signal has been chosen to be greater than $200 \mathrm{~Hz}$ in order to reduce as much as possible the effects of the electrohydrodynamic instabilities [9]. This frequency also avoids the occurrence of undesired AC modulation. The amplitude has been only represented for three voltages $(3.5 \mathrm{~V}, 5 \mathrm{~V}, 7 \mathrm{~V})$ to simplify the graphical representation. However, further validation with more voltage states will be presented in section IV.

As can be seen in Fig. 4, the maximum phase error between simulations and measurements is small at each voltage, so that the intermediate states are well modeled using the inhomogeneous tensors $\overline{\overline{\varepsilon_{r}}}\left(z, V, f_{R F}\right)$. The maximum phase difference between measurements and simulations in the band from 96 to $104 \mathrm{GHz}$ is represented in Fig. 5 as a function of the voltage (black curve). Different sources of errors have been evaluated. Assuming reasonable convergence criteria, the numerical error in the method to calculate $\theta(\mathrm{z})$ has been evaluated and is smaller than $0.7^{\circ}$, whereas the error produced by the convergence of MoM (number of layer and meshing accuracy) is smaller than $0.5^{\circ}$. Therefore the errors can mostly be attributed to the manufacturing tolerances.

In the simulations of the curves represented in Fig. 4, segmentation of the tensors $\overline{\overline{\varepsilon_{r}}}\left(z, V, f_{R F}\right)$ into a number of layers $\mathrm{N}_{\mathrm{L}}(\mathrm{V})$, which is the number of layers of the stratified medium, has been considered at each voltage. The segmentation process has been defined at each voltage (V) by using the tilt angles shown in Fig. 2, $\theta(z, V)$. The process is initiated by selecting the center of the cell $\left(\mathrm{z}=\mathrm{h}_{\mathrm{lc}} / 2\right)$ and by taking the parameter $\Delta \theta$ to obtain the angular range that defines a layer. Then, the ordinate axis is divided into constant intervals of $\Delta \theta$, and the points at the abscissa axis will provide the interfaces that define the layers. The permittivity tensor in each layer is calculated by 


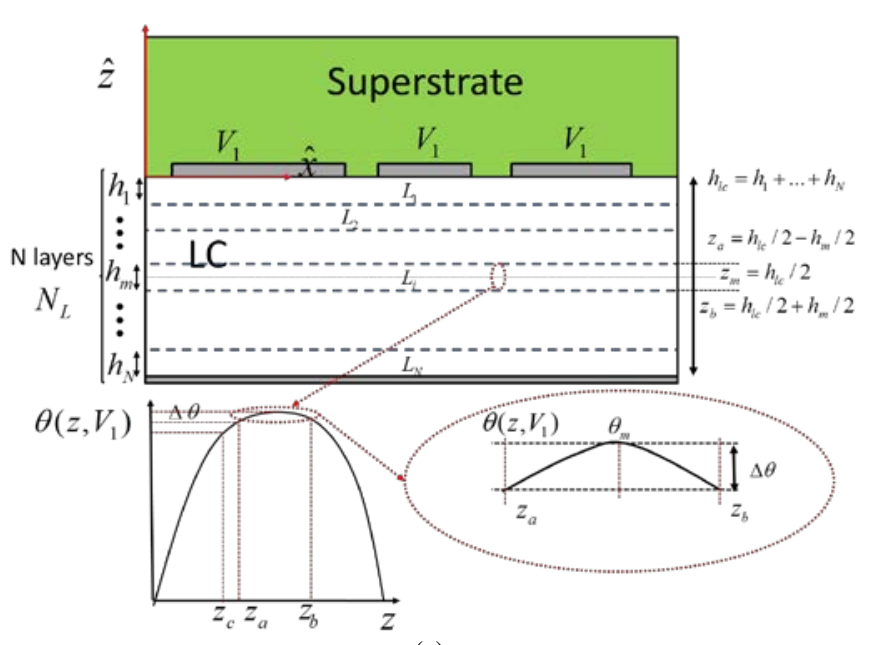

(a)
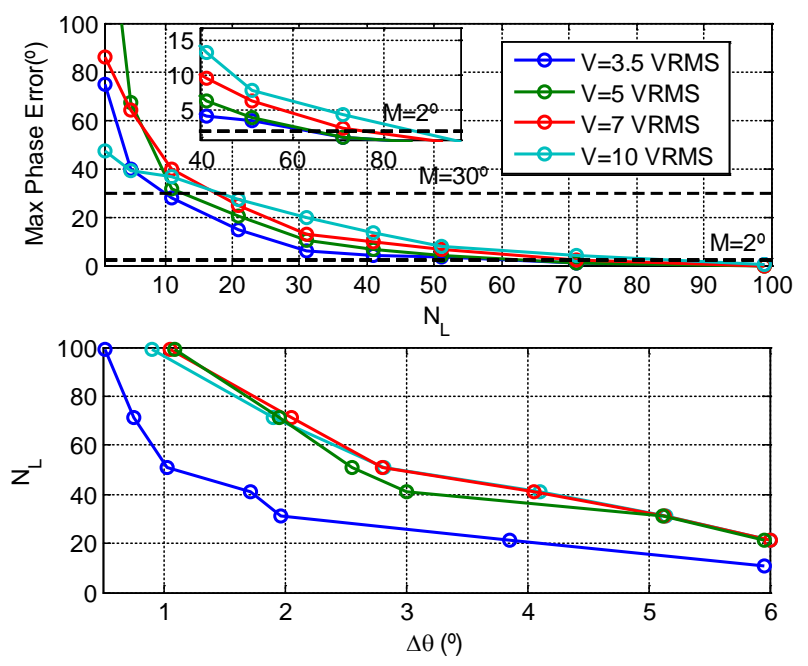

(b)

Fig. 6. (a) LC segmentation of a reflectarray cell. (b) Convergence curves of the cell of Fig. 4. Maximum Phase Error and Angular Parameter $\Delta \theta$ versus the Number of Layers, $\mathrm{N}_{\mathrm{L}}$

using the angular value at its center (Fig. 6 (a)). Note that the thicknesses of the resulting layers, which are symmetric with respect to the center of the cell, depend on the corresponding angular curve and $\Delta \theta$, so that they will not be constant. Note also that generally the last two layers do not have the same angular increment as the others, although the angular variation of these is in the range defined by $\Delta \theta$.

The value of $\Delta \theta$ should be chosen to be small because it is useful to model certain homogeneous regions in the curves $\theta(z, V)$, which are usually produced at the higher voltage states (see Fig. 2). However, $\Delta \theta$, which is related with the number of layers $\mathrm{N}_{\mathrm{L}}$, generally depends on the cell geometry, the voltage state, and the required accuracy to calculate the reflection coefficient, so that if the chosen value of $\Delta \theta$ is not small enough to calculate the reflection coefficient with good accuracy (mainly in the phase), its value should be reduced. Therefore, an iterative convergence analysis must be made in which the phase accuracy is calculated at several values of $\Delta \theta$. In this process, the accuracy can be evaluated by calculating the error resulting from the phase provided by the corresponding segmentation and a phase reference. This latter is calculated by using a large number of layers and the same segmentation, and whose error is considered to be zero. This evaluation must be repeated at several values of $\Delta \theta$ until a certain phase error threshold (M) is achieved.

Using these considerations, a convergence analysis has been made prior to obtain the curves of Fig. 4; the results are plotted in the Fig. 6 (b). In this figure, the maximum phase error (from 96 to $104 \mathrm{GHz}$ ) as function of the number of segmentation layers, $\mathrm{N}_{\mathrm{L}}$, is represented at several voltages for the cell considered, using a reference phase associated with 100 layers. The value of $\Delta \theta$ and its correspondence with the number of layer is also plotted. The results show that more than 80 layers should be used to obtain $\pm 2^{\circ}$ phase error, whereas a value of $\Delta \theta=1$ is adequate to obtain convergence for practically all voltages with around $\mathrm{M}=2^{\circ}$. This value was selected to obtain the curves represented in Fig. 4. Fig. 6(b) also shows that convergence is achieved with a different number of layers for each voltage. Segmenting the LC into a larger number of layers is required when the voltage increases, thus indicating that the rising and falling regions of $\theta(z, V)$ must be modeled with a large number of layers.

Note that since the cells will be part of a complete antenna (as explained in Section IV), the convergence analysis should be done at each cell of the reflectarray. However, this process is computationally intensive, so that the result presented in Fig. 6 (b) could be reasonably generalized to all the elements that form the antenna.

It should be also mentioned that the value of $\mathrm{M}$ depends on the application, the antenna configuration and the radiation pattern to be configured. In a dual reflector configuration in which the LC-reflectarray acts as a sub-reflector to obtain beam-scanning in one dimension, the phase errors on the reflectarray surface (as a consequence of the tolerances, the effect of the LC, etc) become periodic because the required phase-shift is progressive. This produces a specular beam [23], which in turn is amplified by the main reflector producing undesired sidelobes if the phase errors are above $\pm 20^{\circ}$. In single-offset configuration, phase errors above $\pm 25^{\circ}$ affect the side-lobes and the cross-polar levels of a shaped beam [24], whereas that a value of $M= \pm 30^{\circ}$ is usually tolerable to generate a collimated beam. However even in the best case, it is necessary to use at least 20 layers to model the LC (see Fig. 5(b)), making the electromagnetic simulations and the extraction of the voltage dependence of the cells computationally inefficient. If numerical methods of volume decomposition are used, the problem is particularly inefficient, especially if the number of layers is relatively large due to the meshing necessary to describe the very thin layers resulting after the segmentation. In the case of SD-MoM, convergence problems appear in respect to the number of Floquet's harmonics and basis functions needed to obtain an accurate solution, which rises with the number of layers especially if these are thin, thus increasing the computation time. As an example, the computation of the reflection coefficient at a certain angle of incidence, with the LC segmented into 20 layers for the voltage V=7 VRMS, requires a computation time (32 bits Intel Core Duo $2.66 \mathrm{GHz}$ 
processor and 4 GB RAM) of 14 s/freq using SD-MoM and 992 s/freq using FIT (Finite Integration Technique). CST [25] was used to evaluate this case.

\section{B. Extraction by using an Effective Homogeneous Tensor}

To reduce the computational time needed to analyze the stratified media, the use of an effective homogeneous tensor, $\overline{\overline{\varepsilon_{r}}}\left(V, f_{R F}\right)$, is proposed.

To properly define the effective permittivity tensor of a reflectarray cell, it is necessary to analyze the cells first by using very accurate modeling, and then to use the calculated electrical parameters to extract the homogeneous permittivity. Note the use of an effective homogeneous tensor is appropriate for the structure shown in Fig. 3, because it can be demonstrated that each one of the possible values of the homogeneous director, $\hat{n}(\theta(V))$, is able to represent the phase in the entire band at a certain state of voltage. Otherwise, these tensors could not be defined. This can be seen in Fig. 7, in which the phase of the cell shown in Fig. 4 is plotted at $\quad 100 \mathrm{GHz}$ as a function of the angular parameter, $\theta$, which represents all the possible homogeneous directors $(\hat{n}=\cos (\theta) \hat{y}+\sin (\theta) \hat{z})$. Therefore, one possibility to obtain an accurate effective permittivity at a certain voltage and frequency consists of sweeping the value of $\theta$, until the phase error is low enough compared with that provided by accurate modeling, thus obtaining $\theta_{e f f}\left(V, f_{R F}\right)$ and $\hat{n}\left(\theta_{\text {eff }}\right)$.

Although this strategy provides models from which the electromagnetic behavior of the cells can be predicted with very small error, it would require the previous calculation by using an accurate approach, so that this procedure is not an alternative, but is an additional step to the stratified media modeling. To obtain an efficient alternative that does not require the use of an accurate approach (3D model, stratified media), the use of an effective tensor resulting from a spatial average is proposed.

For cells fulfilling $\theta_{p 0}=\theta_{p d}$, this tensor is defined from the average tilt angle at each voltage, $\theta_{a}(V)$, which can be obtained by:

$$
\theta_{a}(V)=f(\theta(V, z))=\frac{1}{d} \int_{z=0}^{z=d} \theta(V, z) d z
$$

Once $\theta_{a}(V)$ and $\hat{n}_{a}(V)$ have been calculated, the effective tensor $\overline{\overline{\varepsilon_{r a}}}\left(\theta_{a}(V), f_{R F}\right)$ at each voltage and frequency can be easily deduced by using (1).

Equation (6) is an approach that allows improvements to the efficiency of the calculation of the voltage dependence of the cells but at expense of assuming errors, since the effect of the inhomogeneity tensor on the RF electric field is only averaged. Therefore, these errors must be evaluated.

Note that since the phase curve with respect to the angular value $\theta$ is smooth (Fig. 7), the errors of assuming the approach (6) are expected to be small. The errors will be worse as the electric field becomes less uniform in the cell, which is produced at the intermediate states (see Fig. 2). At the states near the saturation, uniformity is produced practically throughout the entire

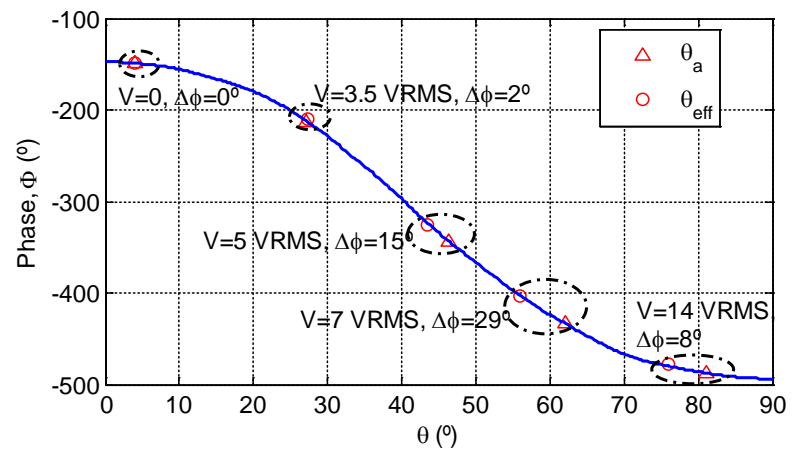

Fig. 7. Phase of the reflection coefficient of the cell of Fig. 4 as function of the angle $\theta$, which represents all the possible homogeneous directors $(\hat{n}=$ $\cos (\theta) \hat{y}+\sin (\theta) \hat{z})$. Frequency: $100 \mathrm{GHz}$. Angle of incidence: $\theta_{\text {in }}=$ $45^{\circ}, \varphi_{\text {in }}=90^{\circ}$. Polarization: TM.

volume, similarly to the extreme states. Thus, since the phase variation is low at angular values greater than $\theta=70^{\circ}$ (see Fig. 7 ), it is expected that the maximum error in the modeling occurs at the states for which $40^{\circ} \leq \theta \leq 70^{\circ}$.

It should be mentioned that several functions, $f(\theta(V, z))$, have been checked to transform the angular distribution $\theta(V, z)$ into $\theta_{a}(V)$, and the one that provides the lowest phase error with respect to the stratified media results for several cases is the average (6). The function $f(\theta(V, z))$ must increase with $\mathrm{V}$ and also satisfy the condition that the transformed angles at the repose $(\mathrm{V}=0)$ and at infinite voltage are the pretilted, $\theta_{p}$, and $90^{\circ}$, respectively.

Fig. 4 shows the amplitude and phase of the reflection coefficient as a function of frequency calculated using the effective homogeneous tensors, $\overline{\overline{\varepsilon_{r a}}}\left(\theta_{a}(V), f_{R F}\right)$, associated with the same bias voltages that were previously considered in Sec. III. A. These tensors correspond to the average angles: $\theta_{a}=\left(4^{\circ}, 27^{\circ}, 47^{\circ}, 62^{\circ}, 81^{\circ}\right)$.

Note that the tensors associated with the angles, $\theta_{a}=4^{\mathrm{o}}$ and $\theta_{a}=81^{\circ}$, provide practically the same phase state as those for the angle $\theta_{a}=0^{\circ}$ and $\theta_{a}=90^{\circ}$, which were used in [8] assuming zero pretilt angles and an infinite voltage for the maximum biasing state, respectively, as an approximations. Thus, the RMS voltage around $14 \mathrm{~V}$ could be considered as the saturation voltage for the LC based cell. However, a slight reduction of the phase range is produced as a consequence of the pretilt angle $\left(\theta_{p(0, d)}=4^{\circ} \Rightarrow \theta_{a}=4^{\circ}\right.$ at $\left.V=0\right)$, which besides affecting the curves in Fig. 2, also affects the phase range so that this latter increases as the pretilt is higher. Thus, the use of polyimide films that provide pretilt angles between $1^{\circ}$ and $4^{\circ}$ is recommendable.

As can be seen in Fig. 4, the measured amplitude and phase curves fit well between both, the rigorous model represented by $\overline{\overline{\varepsilon_{r}}}\left(z, V, f_{R F}\right)$ and the model that uses the effective tensors $\overline{\overline{\varepsilon_{r a}}}\left(\theta_{a}(V), f_{R F}\right)$, although some errors are obtained as expected. Fig. 5(a) shows the maximum phase difference between the two numerical models (green curve) for various voltage states in the band from 96 to $104 \mathrm{GHz}$, whereas that Fig. 7 shows this error represented at several values of the angular parameter, $\theta$, at 100 GHz. In this case, the values $\theta_{\text {eff }}=\left(4^{\circ}, 27^{\circ}, 44^{\circ}, 56^{\circ}, 77^{\circ}\right)$ 


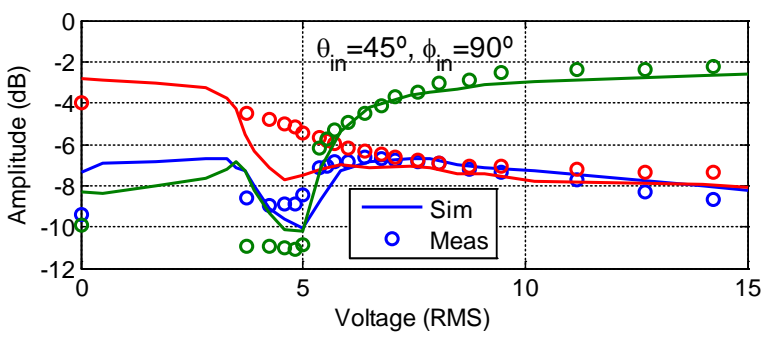

(a)

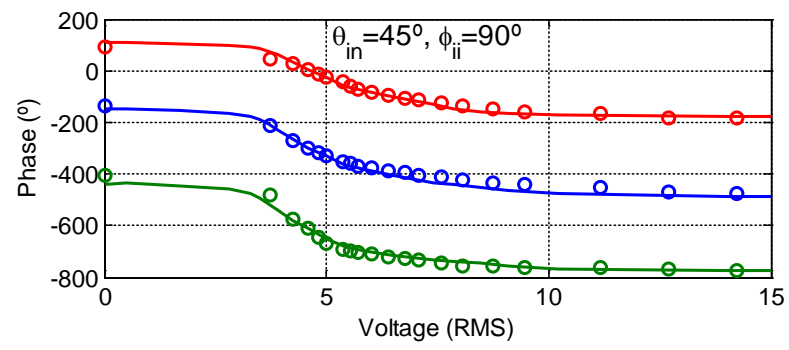

(b)

Fig. 8. Simulated and measured amplitude (a) and phase (b) of the reflection coefficient as a function of the applied RMS Voltage of the cell of Fig. 4 at three frequencies: $96 \mathrm{GHz}$ (red curve), $100 \mathrm{GHz}$ (blue curve) and $104 \mathrm{GHz}$ (green curve). Biasing signal: $1 \mathrm{kHz}-$ sine. Angle of incidence, $\theta_{\text {in }}=45^{\circ}$, $\varphi_{\text {in }}=90^{\circ}$.Polarization: $\mathrm{TM}$

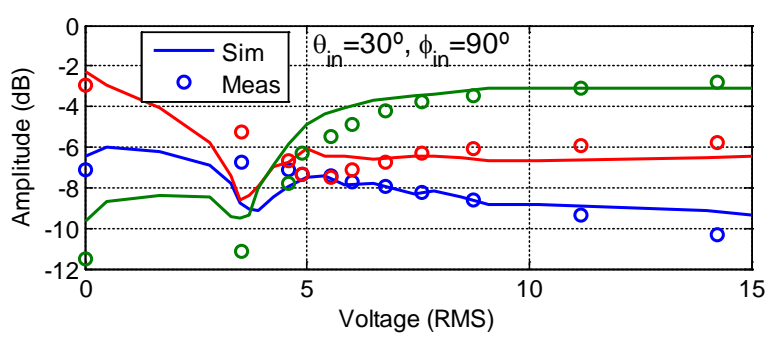

(a)

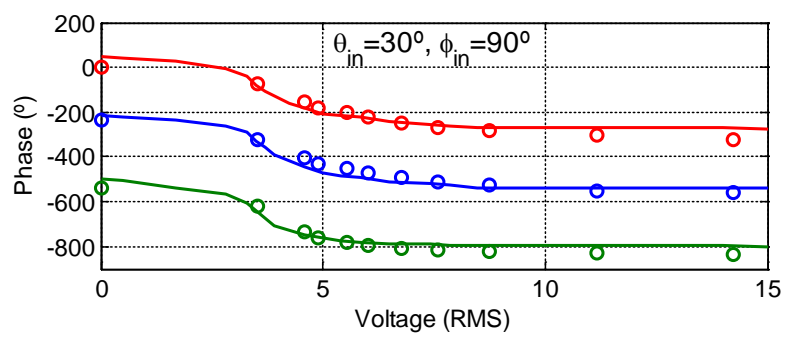

(b)

Fig. 9. Simulated and measured amplitude (a) and phase (b) of the reflection coefficient as a function of the applied RMS Voltage of the cell of Fig. 4 at three frequencies: $96 \mathrm{GHz}$ (red curve), $100 \mathrm{GHz}$ (blue curve) and $104 \mathrm{GHz}$ (green curve). Biasing signal: $1 \mathrm{kHz}$-sine. Angle of incidence, $\theta_{\text {in }}=30^{\circ}, \varphi_{\text {in }}=90^{\circ}$. Polarization: TM

were extracted from the simulations of the stratified media approach by using the procedure described in the beginning of the section III. B.

As is noted, the effective tensors $\overline{\overline{\varepsilon_{r a}}}\left(\theta_{a}(V), f_{R F}\right)$ describe very well the phase response for the lower and higher voltage states, for which phase differences of around $5^{\circ}-10^{\circ}$ are obtained.
However, the phase difference increases up $32^{\circ}$ at the voltages between $8 \mathrm{~V}$ and $10 \mathrm{~V}$. Note that although the phase difference increases up $32^{\circ}$, the average error of all the states is around $15^{\circ}$, which is acceptable. For applications requiring more accuracy, the stratified media modeling becomes necessary.

The phase errors due to the approach (6) depends on the cell structure, since it further determines the RF electric field distribution in the cell, and therefore its phase variation. Although it is expected that these errors are assumable in most cell topologies, especially if the phase curve is smooth, the convenience of using (6) should be evaluated for other cells.

The evaluation may be carried out by selecting a value of the angle, $\theta$, for which the error is estimated to be maximal $\left(50^{\circ} \leq \theta\right.$ $\leq 70^{\circ}$ ), and then find the curve in Fig. 2 for which the spatial average, $\theta_{\mathrm{a}}$, matches with the selected angle. This curve must be used to evaluate the phase using the stratified media approach and the average error. If this latter is assumable, the approach given by (6) can be used in the design procedure of the antenna (Section IV). This provides an efficient alternative because the effective tensors reduces the problems to that of one layer, thus improving the simulation time at each voltage with respect to the inhomogeneous modeling. Using the example considered above to evaluate the computational efficiency, it has been shown that the time to analyze the cell considered in the paper ( $\mathrm{V}=7$ VRMS and 20 layers) is reduced by a factor of 11 for SD-MoM, and 17 for FIT.

\section{Complete RF Design of A LC-BAsed ReflectarRay ANTENNA}

Since the tensors $\left.\overline{\overline{\varepsilon_{r}}}\left(z, V, f_{R F}\right)\right)$ and $\overline{\overline{\varepsilon_{r a}}}\left(\theta_{a}(V), f_{R F}\right)$ are able to model the intermediate states of the cells, these allow the calculation of the amplitude and phase curves versus the voltage, which are needed to design LC-based reconfigurable reflectarray antennas. These results are plotted in Figs 8 and 9 for the cell considered in the paper, in which the simulated and measured amplitude (a) and phase (b) of the reflection coefficient as a function of the voltage are shown at three frequencies (96, 100 and $104 \mathrm{GHz}$ ) and at two different angles of incidence: $\theta_{\text {in }}=45^{\circ}, \varphi_{\text {in }}=90^{\circ}$ and $\theta_{\text {in }}=30^{\circ}, \varphi_{\text {in }}=90^{\circ}$, respectively. The approach used to calculate these curves is $\left.\overline{\overline{\varepsilon_{r}}}\left(z, V, f_{R F}\right)\right)$, whereas the measurements were done in a periodical environment [8], as aforementioned. As can be seen, the voltage dependence of the cell can be well predicted.

It should be mentioned that the RF design of a complete reflectarray antenna based on LC can be divided into two steps: the design of the antenna geometry to obtain a certain electrical performance, and the design of a certain radiation pattern. The first step is described in [8], which uses only the extreme states and the concept of isotropic effective permittivity to obtain good computational efficiency in the design procedure. Once the design data of all the cells are obtained (sizes of conductor elements, LC and superstrate thicknesses, and superstrate permittivity), the next step consists of designing the required radiation pattern, for which the voltage dependence of the reflection coefficient must be known. The most important contribution of the two techniques proposed in this paper is that the phase-voltage curves for a given reflectarray cell can be 
obtained through simulations and not from measurements. Thus, the voltage dependence can be calculated at each cell taking into account the corresponding dimensions, angle of incidence and frequency (note that the conductive elements can be different in each cell even though the antenna is reconfigurable). It should be pointed out that although the modeling of the intermediate states described here can be used directly in the design procedure of the cells in order to obtain more refined designs, the procedure that considers only the extreme states provides good results to design the cells. Thus, the high computational cost that results from the simulation of the intermediate states is assumed in an analysis process, but not in a design procedure.

Once the data acquisition has been done, it should be stored in a look-up table from which the corresponding voltage to be applied at each cell is determined each time a required radiation pattern (determined by the phase-shift distribution on the reflectarray) must be configured.

\section{CONCLUSIONS}

Two strategies to model the LC for each biasing voltage in reflectarray cells, and therefore to predict the voltage dependent reflection amplitude and phase have been presented, thus providing useful tools for practical implementations. The most rigorous approach models the LC as a stratified media along $\mathrm{z}$ to represent the inhomogeneous tensor, which is obtained by solving the elastic problem associated with biasing of the LC. This method permits a lower level of error to be obtained in the computation of the reflection coefficient of the reflectarray cells, but at the expense of very high computational times and convergence issues, since a large number of layers are needed (at least 20). The second method is based on an effective homogeneous tensor that is able to model the inhomogeneity of the LC layer, which can be calculated using the average tilt angle along the cell. This latter approach allows the reflectarray cell to be modeled at each voltage state, is computationally more stable and gives a relatively small phase-error.

\section{ACKNOWLEDGMENT}

The authors are grateful to Mr Atsutaka Manabe of Merck KGaA, Darmstadt, Germany for fruitful discussions and for supplying the liquid crystal materials.

\section{REFERENCES}

[1] W. Hu, R. Dickie, R. Cahill, H. Gamble, Y. Ismail, V. Fusco, D. Linton, N. Grant, and S. Rea, "Liquid Crystal Tunable mm Wave Frequency Selective Surface,” IEEE Microw Wireless. Comp. Lett., vol. 17, no. 9, pp. 667-669, Sep. 2007.

[2] O. H, Karabey, S. Bildik, S. Bausch, S. Strunck, A. Gaebler, and R. Jakoby, "Continuously Polarization Agile Antenna by Using Liquid Crystal-Based Tunable Variable Delay Lines,” IEEE Trans. Antennas Propagat., vol. 61, no. 1, pp. 70-76, Jan. 2013.

[3] M. Yazdanpanahi and D. Mirshekar-Syahkal, "Millimeter-wave liquidcrystal-based tunable bandpass filter" in Proc. IEEE Radio and Wireless Symposium (RWS), Santa Clara, USA, Jan 2012.

[4] S. Christie, R. Cahill, N. Mitchell, Y. Munro, and A. Manabe, "Electronically Scanned Rotman Lens Antenna with Liquid Crystal Phase Shifters,” IET Electron Lett., vol. 49, no. 7, pp. 445-447, Mar. 2013.
[5] W. Hu, R. Cahill, J. A. Encinar, R. Dickie, H. Gamble, V. Fusco, and N. Grant, "Design and Measurement of Reconfigurable Millimeter Wave Reflectarray Cells With Nematic Liquid Crystal,” IEEE Trans. Antennas Propagat., vol. 56, no. 10, pp. 3112-3117, Oct. 2008.

[6] A. Moessinger, R. Marin, S. Mueller, J. Freese and R. Jakoby, "Electronically reconfigurable reflectarray with nematic liquid crystal," Electron. Lett., vol. 42, no. 16, pp. 899-900, Aug, 2006.

[7] W. Hu, M. Y. Ismail, R. Cahill, J. A. Encinar, V. Fusco, H. Gamble,, D. Linton, R. Dickie, N. Grant, and S. P. Rea, "Liquid-crystal-based reflectarray antenna with electronically switchable monopulse patterns," Electron. Lett., vol. 43, no. 14, pp. 744-745, Jul, 2007.

[8] G. Perez-Palomino, P. Baine, R. Dickie, M. Bain, J. A. Encinar, R. Cahill, M. Barba, and G. Toso, "Design and Experimental Validation of Liquid Crystal-Based Reconfigurable Reflectarray Elements With Improved Bandwidth in F-Band," IEEE Trans. Antennas Propagat., vol. 61, no. 4, pp. 1704-1713, Apr. 2013.

[9] P.G. de Gennes and J. Prost, The Physics of Liquid Crystals. 2nd ed. Clarendon Press, 1995.

[10] F. A. Fernández, S. E. Day, P. Trwoga, H. Deng, and R. James,“Threedimensional dynamic modelling of liquid crystal display cells using finite elements,” Mol. Cryst Liq. Cryst, vol. 375, pp. 291-299, 2002.

[11] R. James, F. A. Fernandez, S. E. Day, S. Bulja, and D. Mirshekar-Syahkal, "Accurate Modeling for Wideband Characterization of Nematic Liquid Crystals for Microwave Applications,", IEEE Trans. Microw. Theory Tech., vol. 57, no. 12, pp. 3293-3297, Dec. 2009.

[12] K. R. Welford and J. R. Sambles, "Analysis of electric field induced deformation in a liquid crystal for any applied field", Mol. Cryst. Liq. Cryst. vol. 147, no. 1, pp. 25-42, 1987.

[13] J. Nehring, A. R. Kmetz, and T. J. Scheffer, "Analysis of weak-boundarycoupling effects in liquid-crystal displays,” J. Appl. Phys., vol. 47, no 3, pp. 850-858, 1976.

[14] X. Nie, H. Xianyu, R. Lu, T. X. Wu, and S. T. Wu, "Pretilt Angle Effects on Liquid Crystal Response Time,” IEEE J. Display Technol., vol. 3, no. 3, pp. 280-283, Sep. 2007.

[15] R. Dickie, et al., "Electrical characterisation of liquid crystals at millimetre wavelengths using frequency selective surfaces" Electron Lett., vol. 48, no. 11, pp. 611-612, May. 2012.

[16] G. Perez-Palomino, J. A. Encinar, M. Barba, and E. Carrasco, "Design and Evaluation of Multi-Resonant Unit-Cells Based on Liquid Crystals for Reconfigurable Reflectarrays," IET Microw. Antennas Propag., vol. 6, no. 3, pp. 348-354, Feb. 2012.

[17] G. Perez-Palomino, J. A. Encinar, and M. Barba, "Accurate Electromagnetic Modeling of Liquid Crystal Cells for Reconfigurable Reflectarrays,” in Proc. 5th Eur. Conf. on Antennas Propag,, Rome, Italy, pp. 997-1001, April. 2011.

[18] K. K. Karnati, Y. Yusuf, S. Ebadi, and Xun Gong, "Theoretical Analysis on Reflection Properties of Reflectarray Unit Cells Using Quality Factors,” IEEE Trans. Antennas Propagat., vol. 61, no. 1, pp.201-210, Jan. 2013.

[19] R. Mittra, C. H. Chan, and T. Cwik., "Techniques for Analizing Frequency Selective Surfaces- A Review” Proc IEEE., vol. 76, no. 12, pp. 1593-1615, Dec. 1988.

[20] C. M. Krowne, "Fourier Transformed Finding Propagation Matrix Method of Characteristics of Complex Anisotropic Layered Media," IEEE Trans. Microw. Theory Tech., vol. 32, no. 12, pp. 1617-1625, Dec. 1984.

[21] F. L. Mesa, R. Marques, and M. Horno., "A general algorithm for computing the bidimensional spectral Green's dyad in multilayered complex bianisotropic media: the equivalent boundary method" IEEE Trans. Microw Theory Tech., vol. 39, no. 9, pp. 1640-1649, Sep. 1991.

[22] R. R Boix, N. G. Alexopóulos, and M. Horno., "Efficient numerical computation of the spectral transverse dyadic Green's function in stratified anisotropic media" J Electromagnet Wave., vol. 10, no. 8, pp. 1047-1083, 1996.

[23] R. El Hani, and J. J. Laurin, "Specular Reflection Analysis for OffSpecular Reflectarray Antennas,” IEEE Trans. Antennas Propagat., vol. 61, no. 7, pp. 3575-3581, Jul. 2013.

[24] J. A. Encinar, M. Arrebola, L. F. de la Fuente, and G. Toso, “A TransmitReceive Reflectarray Antenna for Direct Broadcast Satellite Applications," IEEE Trans.Antennas Propagat., vol. 59, no. 9, pp. 32553264, Apr. 2011

[25] CST Microwave Studio. [Online]. Available: http://www.cst.com. 


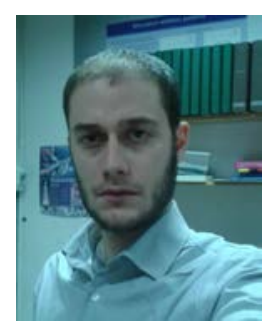

Gerardo Perez-Palomino was born in Granada, Spain. He received the Telecommunications Engineer degree in 2009, and the M.S. degree in Communications Technologies and Systems in 2010, both from the Universidad Politécnica de Madrid (UPM), Spain, where he is currently pursuing the Ph.D. degree after being awarded a Ph.D. Scholarship from the Spanish "Ministerio de Ciencia e Innovación.”

Since 2008, he has been working with the Applied Electromagnetism and Microwaves Group at UPM as a Research Assistant. His research interests include the analysis, characterization, modelling and design of antenna and microwave circuits, and the electromagnetic theory. He is involved in the development of reconfigurable antennas in millimeter and sub-millimeter wavelengths and software tools for designing reflectarrays.

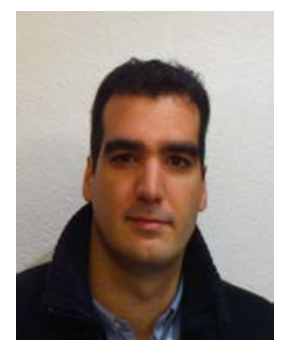

Rafael Florencio received the Licenciado degree in physics from the University of Seville, Seville, Spain, in 2008, and the master's degree in Communications Technology and Systems from the Polytechnic University of Madrid, Madrid, Spain, in 2010.

In 2010, he joined the Electronics and Electromagnetism Department, University of Seville, where he is currently working towards the Ph.D.

Mr. Florencio was the recipient of a Scholarship financed by the Junta de Andalucía.

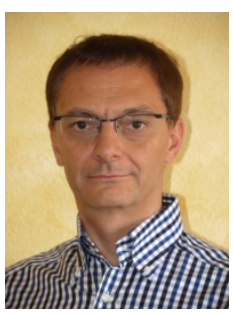

José A. Encinar (S'81-M'86-SM'09-FM'10) was born in Madrid, Spain. He received the Electrical Engineer and Ph.D. degrees, both from Universidad Politécnica de Madrid (UPM), in 1979 and 1985, respectively.

Since January 1980 has been with the Applied Electromagnetism and Microwaves Group at UPM, as a Teaching and Research Assistant from 1980 to 1982, as an Assistant Professor from 1983 to 1986, and as Associate Professor from 1986 to 1991. From February to October of 1987 he stayed at Polytechnic University, Brooklyn, NY, as a Post-doctoral Fellow of the NATO Science Program. Since 1991 he is a Professor of the Electromagnetism and Circuit Theory Department at UPM. He was with the Laboratory of Electromagnetics and Acoustics at Ecole Polytechnique Fédérale de Lausanne (EPFL), Switzerland in 1996, and with the Institute of Electronics, Communication and Information Technology (ECIT), Queen's University Belfast, U.K. in 2006 and 2011, as a Visiting Professor. His research interests include numerical techniques for the analysis of multi-layer periodic structures, design of frequency selective surfaces, printed arrays and reflectarrays.

Prof. Encinar has published more than one hundred and fifty journal and conference papers, and he is holder of five patents on array and reflectarray antennas. He was a co-recipient of the 2005 H. A. Wheeler Applications Prize Paper Award and the 2007 S. A. Schelkunoff Transactions Prize Paper Award, given by IEEE Antennas and Propagation Society. He has been a member of the Technical Programme Comity of several International Conferences (European Conference on Antennas and Propagation, ESA Antenna Workshops, Loughborough Antennas \& Propagation Conference). He is an IEEE Fellow member.

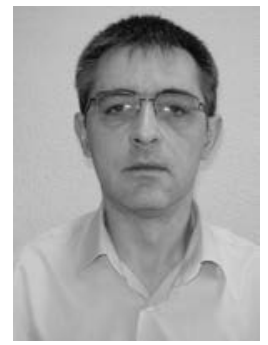

Mariano Barba (M’09) was born in Murcia, Spain. He received the Ingeniero de Telecomunicación and Ph.D. degrees from the Universidad Politécnica de Madrid, Madrid (UPM), Spain, in 1990 and 1996, respectively. From 1991 to 1994 he was with the Depto. de Electromagnetismo y Teoría de Circuitos (UPM) as postgraduate researcher. During this time, he developed numerical methods and algorithms to characterize and design microwave passive devices. From 1994 to 2002, he has been involved in the R\&D departments of several companies and institutions developing antennas and microwave circuits for space and terrestrial communication applications. In 2002, he joined to the "Depto. de Electromagnetismo y Teoría de Circuitos", (UPM) as Associate Professor. His research interests include the analysis, characterization and design of antenna and microwave circuits. He is currently involved in the development and design of multibeam and reconfigurable antennas.

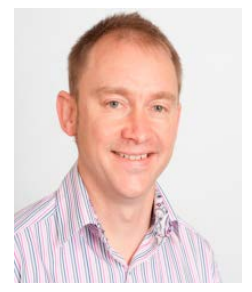

Raymond Dickie received a BEng honours degree and $\mathrm{PhD}$ in Electrical and Electronic Engineering from the Queen's University Belfast in 2001 and 2004 respectively.

In October 2004 joined the high frequency electronic circuits and antennas group at The Institute of Electronics, Communications and Information Technology (ECIT) in Belfast where he is now employed as a senior engineer working on mm-wave components. His work on freestanding frequency selective surfaces has been patented and includes fabrication methods using SOI, metal and polymer mesh technology. Dr Dickie has significant experience in photolithographic processing including thick positive and negative photoresist, RIE of polymers and oxides, DRIE of silicon, CVD metal deposition, high conductivity stress controlled electroplating, and SEM imaging methods. He is experienced in working in clean room environments where he develops MEMS devices. Dr Dickie has co authored over 40 publications, his high frequency research interests include numerical modelling of high frequency structures and precision quasi-optical measurements in the millimeter and sub millimeter wave bands.

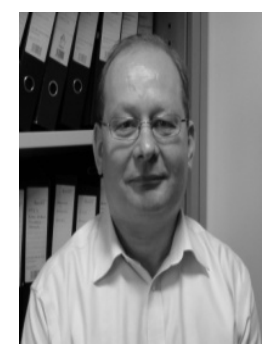

Robert Cahill (M'10-SM'11) received a BSc (1st class) honours degree in Physics from the University of Aston in Birmingham in 1979 and a PhD degree in microwave electronics from the University of Kent at Canterbury in 1982.

He joined Queen's University Belfast (QUB) in 1999 after a 17 year career working in the UK space and defence industry, where he worked on antenna and passive microwave device technology projects. During this time he pioneered methods for predicting the performance of antennas on complex scattering surfaces such as satellites and has developed techniques for analysing and fabricating $\mathrm{mm}$ and sub-mm wave quasi-optical dichroic filters. Recently he has established a $100-700 \mathrm{GHz}$ quasi-optical S parameter measurement facility at QUB. He has exploited the results of numerous research projects, sponsored by the European Space Agency, EADS Astrium Space Ltd, the British National Space Agency, the Centre for Earth Observation Instrumentation (CEOI) and the UK Meteorological Office, to develop quasi-optical demultiplexers for atmospheric sounding radiometers in the range $89-500 \mathrm{GHz}$. These include AMSU-B, AMAS, MARSCHALS and the ESA 500GHz demonstrator. Dr Cahill's recent interests also include the characterisation of liquid crystal materials at microwave and mm wavelengths, and strategies for broad banding and creating active reflectarray antennas. He has (co) - authored over 150 publications and holds 4 international patents. 
Paul Baine graduated from the Queen's University Belfast with a first class Beng honours degree in Electrical and Electronic Engineering in 1992. In 1997 he received the $\mathrm{PhD}$ degree for his research in the area of single crystal silicon on glass for display applications. The title of his dissertation was " Fabrication of Thin Single Crystal Silicon Devices on Glass Using Electrostatic Bonding”.

He has been with Queen's University Belfast since 1994 working on research activities involving SOI, Silicon Bonding, the bonding of non standard materials and buried-silicide GPSOI structures and Germanium on Sapphire. More recently he has been involved in LC filled reflectarray fabrication for ESA. He currently holds the role of Manager of the Queen's Advanced MicroEngineering Centre, responsible for the smooth operation of a high class semiconductor and nanotechnology fabrication laboratory as well as providing support for ongoing research contracts. He is research active with over 50 publications to date. Other areas of expertise include: wafer processing technology, photolithography and device fabrication.

Michael Bain received a BSc (Hons) degree in Physics and Electronics from the University of Dundee, Scotland and a Phd from the Queen's University, Belfast, Northern Ireland in 1994 and 2000 respectively.

In November 1998 he joined the Northern Ireland Semiconductor Research Centre (NISRC) at QUB in Belfast where he is now employed as a research fellow. Working on a number of EPSRC funded projects he has gained significant expertise in a variety of bonding techniques and has developed technologies for the integration of metallic layers into SOI substrates. He has had regular access to and received training in the industrial clean-room facilities at Icemos Technologies Belfast, which is a certified standard ISO 9001:2000 company. These include a class ' 1 ' bonding room. He has significant expertise in the deposition and characterisation of PVD and CVD metal layers and has conducted collaborative research with industry on the metallisation of submicron trenches. Dr Michael Bain has considerable experience in MEMS fabrication by surface and bulk micromachining. Using a combination of DRIE, thick resists, aligned bonding and anodic bonding a photoacoustic gas sensor was successfully miniaturised to chip scale. Dr Michael Bain has co-authored over 40 publications.

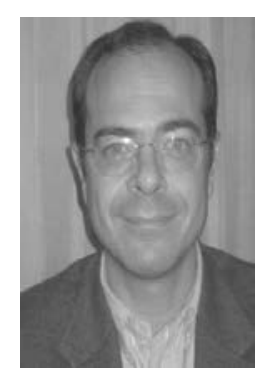

Rafael R. Boix (M'96) received the Licenciado and Doctor degrees in physics from the University of Seville, Seville, Spain, in 1985 and 1990, respectively.

Since 1986, he has been with the Electronics and Electromagnetism Department, University of Seville, where he became Tenured Professor in 2010. His current research interests are focused on the efficient numerical analysis of periodic planar multilayered structures with applications to the design of frequency selective surfaces and reflectarray/transmitarray antennas. 